\title{
Cosmographic study of the universe's specific heat: A landscape for Cosmology?
}

\author{
Orlando Luongo ${ }^{1,2,3}$ and Hernando Quevedo ${ }^{1,4,2 *}$ \\ ${ }^{1}$ Instituto de Ciencias Nucleares, Universidad Nacional Autónoma de México, AP 70543, México, DF 04510, Mexico. \\ ${ }^{2}$ Dipartimento di Scienze Fisiche, Università di Napoli "Federico II", Via Cinthia, I-80126, Napoli, Italy. \\ ${ }^{3}$ INFN Sez. di Napoli, Compl. Univ. Monte S. Angelo Ed. N Via Cinthia, I- 80126, Napoli, Italy. \\ ${ }^{4}$ Dipartimento di Fisica and Icra, Università di Roma "La Sapienza", I-00185 Roma, Italy. \\ ${ }^{5}$ Instituto de Cosmologia, Relatividade e Astrofisica ICRA - CBPF \\ Rua Dr. Xavier Sigaud, 150, CEP 22290-180, Rio de Janeiro, Brazil.
}

\begin{abstract}
We propose a method for constructing the specific heat for the universe by following standard definitions of classical thermodynamics, in a spatially flat homogeneous and isotropic spacetime. We use cosmography to represent the specific heat in terms of measurable quantities, and show that a negative specific heat at constant volume and a zero specific heat at constant pressure are compatible with observational data. We derive the most general cosmological model which is compatible with the values obtained for the specific heat of the universe, and show that it overcomes the fine-tuning and the coincidence problems of the $\Lambda \mathrm{CDM}$ model.
\end{abstract}

PACS numbers:

\section{INTRODUCTION}

All the attempts made to understand the modern cosmological observations indicate the existence of a positive acceleration that has been extensively measured in different surveys [1 [6]. On the other hand, General Relativity predicts a decelerated expansion, if one assumes that the universe is filled by dust only 7 ]. Thus, the universe turns out to be dominated by a new ingredient that is responsible for the cosmic acceleration 8]. Unfortunately, this ingredient forecasts a both surprising and unexpected negative Equation of State (EoS) [9 11]. The physical interpretation of this exotic behavior reflects the need of counterbalancing the gravitational attraction [12. Due to this anomalous behavior, the unknown term to be added in the cosmological puzzle is usually referred to as Dark Energy (DE), filling almost the $70 \%$ of the whole universe [13]. Thence, soon after the discovery of the anomalous dynamics of DE, the former cosmological model was forced to be modified. Thus, thought as the likely most viable explanation, it has been assumed that a cosmological constant $\Lambda$ [14 17] should be included within the Einstein equations. The corresponding model, the so-called $\Lambda$ CDM paradigm, depicts a cold Dark Matter and a late-dominant counterpart represented by the density of $\Lambda$ [18], i.e. $\Omega_{\Lambda}$. Since the model excellently passes all the experimental tests, it strongly entered the modern cosmological convictions, becoming the standard cosmological paradigm [19].

Unfortunately, two profound issues plague the model: the problems of coincidence and of fine-tuning [20]. Particularly, the coincidence points out that an impressive amount of available cosmic data predicts a matter density, $\Omega_{m}$ extremely close to $\Omega_{\Lambda}$. On the other hand, the fine-tuning issue poses several limits on the forecasted cosmological constant and on the measured one. Due to these shortcomings, many other alternative explanations to the problem of DE have been proposed in literature throughout the time [21 25]; however, a self-consistent explanation, both from the theoretical and experimental points of view, seems to be so far out of reach [26].

On the other hand, many efforts have been made recently in order to analyze the thermodynamic properties of cosmological models by using only the geometry of the space of equilibrium states [27, 28] ${ }^{1}$. In particular, it turns out that negative specific heats and non-extensive thermodynamic variables can be used to describe systems with negative EoS's [34]. This kind of systems can be studied further in the context of geometrothermodynamics [27], a formalism that allows one to define and handle non-extensive variables, or by using non-extensive generalizations of the concept of entropy [35].

In this work, we use the standard definitions of classical thermodynamics to define the specific heat at constant volume and at constant pressure on a cosmological background, consisting of the Friedman-Lemaitre-RobertsonWalker spacetime and a perfect fluid as the source of the gravitational field. The formalism of cosmography [36 39 ] is used to represent the specific heat in terms of cosmographic parameters whose values are determined indirectly

\footnotetext{
*Electronic address: orlando.luongo@na.infn.it, quevedo@nucleares.unam.mx

1 A different approach takes into account quantum effects due to fluctuations with particle creation [29-33].
} 
from the observational data for the redshift and the luminosity distance ${ }^{2}$ [41, 42]. In addition, we will relate the thermodynamic properties of the universe [43] with a cosmological model which is able to explain the DE effects, using our thermodynamic assumptions only.

This paper is organized as follows: In Sec. II] we formulate the definition of the specific heat at constant volume and at constant pressure and show that the corresponding thermodynamic potentials are positive as a consequence of the weak energy conditions. In Sec. III we present the main idea of cosmography and evaluate the cosmographic parameters in the case of our universe. Sec. IV] is devoted to the study of the specific heat in terms of the cosmographic parameters. In Sec. $\nabla$, we derive the most general cosmological model that is compatible with the values of the specific heat of the universe. Finally, Sec. VI contains the discussions of our results and suggestions for further research.

\section{SPECIFIC HEAT OF THE UNIVERSE}

To describe the geometric and physical properties of the universe at large scales we assume the validity of the cosmological principle. Then, the geometric properties are determined by the Friedman-Lemaitre-Robertson-Walker (FLRW) line element

$$
d s^{2}=d t^{2}-a(t)^{2}\left[d r^{2}+r^{2}\left(\sin ^{2} \theta d \phi^{2}+d \theta^{2}\right)\right]
$$

where, in accordance with observations, we assume a zero spatial curvature. For a perfect fluid source, the Einstein equations reduce to the Friedman equations

$$
H^{2}=\frac{8 \pi G}{3} \rho_{t}, \quad \dot{H}+H^{2}=-\frac{4 \pi G}{3}\left(3 P+\rho_{t}\right),
$$

where the dots represent the derivative with respect to the cosmic time $t$.

The perfect fluid is the source of the gravitational field of the universe at large scales, and can also be interpreted intuitively as a thermodynamic system [44]. Moreover, it can be shown that the laws of thermodynamics are mathematically consistent with an isotropic and homogeneous geometric background [45]. In fact, using the first law of thermodynamics one can derive an expression for the temperature of the universe whose predictions are in agreement with the observations of the cosmic background radiation [46 49]. Then, following standard definitions of classical thermodynamics [50], we introduce the specific heat

$$
\mathrm{C} \equiv \frac{\partial \hat{U}}{\partial T}
$$

where the energy function $\hat{U}$ determines the type of specific heat. Indeed, according to the standard definitions of internal energy $U$ and enthalpy $h$, the following relations hold

$$
\mathrm{C}_{V}=\frac{\partial U}{\partial T}, \quad \mathrm{C}_{P}=\frac{\partial h}{\partial T}
$$

representing the specific heat at constant pressure $P$ and constant volume $V$, respectively.

For the FLRW model, the energy and enthalpy of the universe can be computed as

$$
U=V_{0} \rho_{t} a^{3}, \quad h=V_{0}\left(\rho_{t}+P\right) a^{3},
$$

where we considered the evolution of the volume up to the first order as $V=V_{0} a^{3}$, with $V_{0}$ being at least proportional to the universe's radius $\frac{1}{H_{0}}$. Furthermore, if we assume the weak energy conditions $\rho_{t} \geq 0$ and $\rho_{t}+P \geq 0$, it follows that $U$ and $h$ are positive quantities ${ }^{3}$. In general, the assumption $V=V_{0} a^{3}$ fulfills the weak energy condition, although other approaches suggest that the volume can be function of the apparent horizon, i.e. $r \propto H^{-1}[51$ ]. The choice $V \propto r^{3}=V_{0} H^{-3}$ would reproduce a causal region with the entropy proportional to $H^{-2}$. However, to characterize the specific heats, we are interested in the small redshift regime, i.e. $z \ll 1$. Thus, under the limits of small $z$, one

\footnotetext{
2 The luminosity distance is not the only observable that can be used to determine the cosmographic parameters. For a different approach see, for instance, [40].

${ }^{3}$ Notice that, despite the total density must be positive, the individual species may be negative, without violating the weak energy conditions.
} 
can first approximate the volume with the simplest choice of $V \propto a^{3}$, without contradicting the causality condition [52].

Our goal is to analyze the time dependence of the specific heat. For comparisons with observational data, however, it is more convenient to use the redshift $z$ which is defined by means of the standard relationships $a=\frac{1}{1+z}$ and $\frac{d z}{d t}=-(1+z) H$. Then, $\mathrm{C}=\frac{d \tilde{U}}{d z}\left(\frac{\partial T}{d z}\right)^{-1}$, and using Eq. (5.5) and the Friedman equations, we obtain

$$
\mathrm{C}_{V}=\frac{1}{T^{\prime}} \frac{d U}{d z}=\frac{3 V_{0} a^{3}}{8 \pi G T^{\prime}}\left(\frac{d H^{2}}{d z}-3 a H^{2}\right),
$$

and

$$
\mathrm{C}_{P}=\frac{1}{T^{\prime}} \frac{d h}{d z}=\frac{1}{T^{\prime}} \frac{d U}{d z}+\frac{V_{0} a^{3}}{T^{\prime}}\left(\frac{d P}{d z}-3 a P\right)
$$

where the prime denotes differentiation with respect to the redshift parameter $z$.

\section{COSMOGRAPHY OF THE UNIVERSE}

The aim of cosmography is the study of the kinematic quantities that characterize the cosmological scenario, without using any particular theory that dictates the dynamics of the gravitational field. For this reason, cosmography is also called cosmokinetics, or kinematics of the universe. To develop the formalism of cosmography, it is necessary to specify only a particular model for the spacetime. If we assume the FLRW model, it is clear that the only the scale factor must be considered. Then, the Taylor expansion of $a(t)$ can be written as follows [53]

$$
a(t)=a_{0}\left[1+H_{0} \Delta t-\frac{q_{0}}{2} H_{0}^{2} \Delta t^{2}+\frac{j_{0}}{6} H_{0}^{3} \Delta t^{3}+\ldots\right]
$$

where the subscript 0 indicates that the corresponding parameters are are evaluated at $z=0$. The deceleration $q$ and jerk $j$ are defined as

$$
q=-\frac{d^{2} a}{d t^{2}}\left(H^{2} a\right)^{-1}, \quad j=\frac{d^{3} a}{d t^{3}}\left(H^{3} a\right)^{-1} .
$$

Alternatively, one can also use the Hubble parameter to obtain

$$
\frac{\dot{H}}{H^{2}}=-(q+1), \quad \frac{\ddot{H}}{H^{3}}=2+j+3 q .
$$

Using Eqs. (8) and (9), it is possible to expand the luminosity distance

$$
d_{l}=(1+z) \int_{0}^{z} \frac{d v}{H(v)},
$$

in terms of the redshift $z$, as follows

$$
d_{L}=\frac{1}{H_{0}}\left[z+\frac{1}{2}\left(1-q_{0}\right) z^{2}+\frac{1}{6}\left(3 q_{0}^{2}+q_{0}-j_{0}-1\right) z^{3}+\ldots\right]
$$

Since the observational data includes the redshift and the luminosity distance, one can now use the cosmographic series (12) to determine the values of the remaining parameters. To this end, we perform a chi-squared analysis, using the most recent Union 2.1 compilation [54]. We first carry out an analysis in which the parameters to be determined, i.e., $H_{0}, q_{0}$ and $j_{0}$, are not confined in any particular interval. In the second analysis, we fix the Hubble constant by using the value obtained from WMAP 7 [54], i.e., $H_{0}=70.2 \mathrm{Km} \mathrm{s}^{-1} \mathrm{Mpc}^{-1}$. The results of the first and the second analysis are presented in Tabs. I and II, respectively.

Notice that the results we obtain for $q_{0}$ are compatible with the results presented in previous works [9, 10]. Moreover, the result for $j_{0}$, suggesting that $j_{0} \sim 1$ [11, 55], is in accordance with the exact theoretical bound predicted by the $\Lambda \mathrm{CDM}$ model. In the next section, we will show that the values of the cosmographic parameters can be used to limit the values of the specific heat. 


\begin{tabular}{|c|c|c|}
\hline$H_{0} \equiv H(z=0)$ & $q_{0} \equiv q(z=0)$ & $j_{0} \equiv j(z=0)$ \\
\hline \hline & & \\
$70.50_{-1.60}^{+1.60}$ & $-0.628_{-0.082}^{+0.085}$ & $0.979_{-0.563}^{+0.531}$ \\
& & \\
\hline \hline
\end{tabular}

TABLE I: Summary of the numerical results obtained by using Eq. (12), with the most recent Supernovae Ia data contained in the Union 2.1 compilation. $H_{0}$ is given in units $s^{-1} K m M_{p c}^{-1}$. For these values we obtain $\chi^{2}=0.975$.

\begin{tabular}{|c|c|c|}
\hline$H_{0} \equiv H(z=0)$ & $q_{0} \equiv q(z=0)$ & $j_{0} \equiv j(z=0)$ \\
\hline \hline $70.30_{-1.40}^{+1.40}$ & $-0.595_{-0.085}^{+0.089}$ & $0.816_{-0.572}^{+0.539}$ \\
& & \\
\hline \hline
\end{tabular}

TABLE II: Summary of the numerical results obtained by using Eq. (12), with the fixed value $H_{0}=(70.30 \pm$ 1.40) $s^{-1} K m M p c^{-1}$, as obtained from WMAP 7 [54]. In this case, $\chi^{2}=0.973$.

\section{COSMOGRAPHIC ANALYSIS OF THE SPECIFIC HEAT}

To find the cosmographic series of the heat capacities, we first rewrite Eqs. (10) as

$$
\frac{d H}{d z}=H \frac{1+q}{1+z}, \quad \frac{d^{2} H}{d z^{2}}=H \frac{j-q^{2}}{(1+z)^{2}},
$$

where we used $a=\frac{1}{1+z}$ and $\frac{d z}{d t}=-H(1+z)$. Moreover, in terms of $z$, the continuity the continuity equation in a FLRW universe can be expressed as

$$
\frac{1}{3} \frac{d \rho_{t}}{d z}=\frac{P+\rho_{t}}{1+z}
$$

Using Eqs. (14) and (2) in the expressions for the specific heats (6) and (7), we finally obtain

$$
\begin{aligned}
& \mathrm{C}_{P}=\frac{V_{0}}{4 \pi G} \frac{H^{2}}{T^{\prime}} \frac{j-1}{(1+z)^{4}}, \\
& \mathrm{C}_{V}=\frac{V_{0}}{8 \pi G} \frac{H^{2}}{T^{\prime}} \frac{2 q-1}{(1+z)^{4}},
\end{aligned}
$$

where we made use of $\frac{d P}{d z}=\frac{H^{2}}{4 \pi G} \frac{j-1}{1+z}$. Eqs. (15) show the direct measurement of the cosmographic parameters $H$, $q$ and $j$, at a given redshift, implies the indirect measurement of the specific heats. In particular, for a measurement at $z=0$, we have that

$$
\begin{aligned}
& \mathrm{C}_{P 0}=\frac{V_{0}}{4 \pi G} \frac{H_{0}^{2}}{T_{0}^{\prime}}\left(j_{0}-1\right), \\
& \mathrm{C}_{V 0}=\frac{V_{0}}{8 \pi G} \frac{H_{0}^{2}}{T_{0}^{\prime}}\left(2 q_{0}-1\right) .
\end{aligned}
$$

First, notice that the sign of $C_{P 0}$ is determined by the sign of the expression $j_{0}-1$. Moreover, we can see that a positive value of $C_{V 0}$ implies a decelerated universe. Therefore, to obtain a universe that is currently in an accelerated phase, we are forced to assume that $C_{V}<0$.

Using the method of standard error propagation, from Eqs. (16) we infer the numerical bounds reported in Tab. III. We see that the results are compatible with a zero value of $C_{P 0}$ and with a negative $C_{V 0}$. These values are also supported by the condition $C_{P}-C_{V}>0$, which is essential to guarantee the stability of a thermodynamical system. Notice that to obtain the numerical values presented in Tab. III we assume that $T^{\prime}=1$. It is clear that $T^{\prime}$ must be 


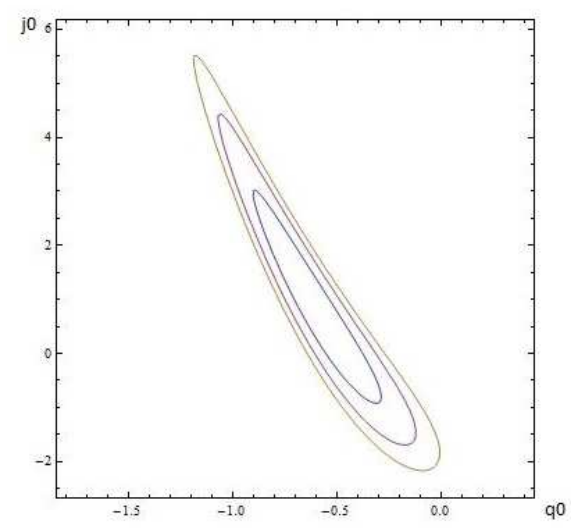

FIG. 1: Contour plots of $j_{0} \mathrm{VS} q_{0}$ at one, two and three sigma of confidence levels for the fitting results of Tab. I.

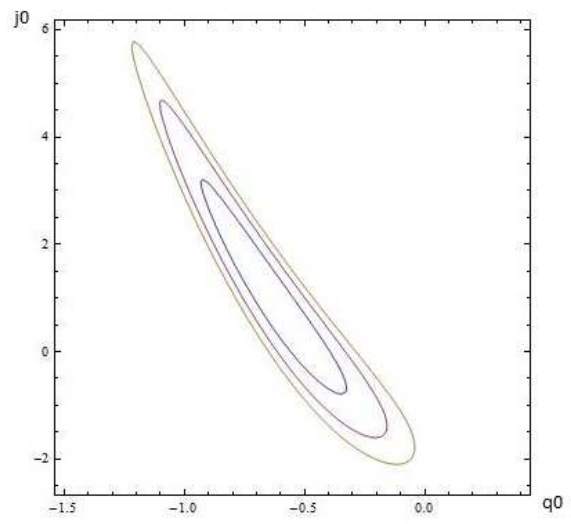

FIG. 2: Contour plots of $j_{0}$ VS $q_{0}$ at one, two and three sigma of confidence levels for the fitting results of Tab. II.

positive to be in agreement with the temperature evolution since the epoch after the big bang. The explicit value of $T^{\prime}>0$ does not affect the main results of our analysis which are a negative value for $\mathrm{C}_{V}$ and a value close to zero for $\mathrm{C}_{P}$.

It is important to mention that the above numerical bounds can be confirmed in the $\Lambda$ CDM model. Indeed, at $z=0$ the $\Lambda \mathrm{CDM}$ model predicts [40]

$$
q_{0 \Lambda C D M}=-1+\frac{3}{2} \Omega_{m}, \quad j_{0 \Lambda C D M}=1 .
$$

Moreover, the jerk turns out to be fixed as $j=1$ for all values of $z$. Consequently, in the range $\Omega_{m}=0.274 \pm 0.015$, $\Lambda \mathrm{CDM}$ predicts that $\mathrm{C}_{P}=0$ and $\mathrm{C}_{V}<0$. Thus, we can conclude that our cosmographic analysis of the specific heat of the universe is in agreement with the predictions of the $\Lambda \mathrm{CDM}$ model. Furthermore, an interesting question arises: Is $\Lambda \mathrm{CDM}$ the most general model that is compatible with the specific heats $\mathrm{C}_{P}=0$ and $\mathrm{C}_{V}<0$ ?

We conclude that the behavior of the specific heat of the universe may suggest insights for understanding the cosmological landscape. In the next section, we will study the consequences of a vanishing $C_{P}$ for all $z$ in the context of relativistic cosmology.

\section{COSMOLOGY WITH VANISHING SPECIFIC HEAT}

According to the results of Secs. III and IV, the present value of the jerk parameter is compatible with $j_{0}=1$. This is in accordance with the cosmological observations and seems to confirm the $\Lambda$ CDM paradigm, which predicts $j=1$ for all the values of the redshift $z$. However, $\Lambda \mathrm{CDM}$ is not the only model that can reproduce this result. Indeed, as a direct consequence of the first of Eqs. (15), the most general perfect fluid, which reproduces the constraint $j=1$, 


\begin{tabular}{|c|c|}
\hline $\mathrm{C}_{P 0} \equiv \mathrm{C}_{P}(z=0)$ & $\mathrm{C}_{V 0} \equiv \mathrm{C}_{V}(z=0)$ \\
\hline \hline & \\
$-0.030_{-0.793}^{+0.748}$ & $-1.587_{-0.151}^{+0.156}$ \\
& \\
\hline \hline
\end{tabular}

TABLE III: Summary of the numerical results obtained from Eq. (16). We used $G=6.67 \times 10^{-11} N m^{2} K g^{-2}, V_{0} \equiv\left(\frac{1}{H_{0}}\right)^{3}$ and conventionally $T^{\prime} \equiv 1 K$. The error propagation is logarithmic. For $q_{0}$ and $j_{0}$ and $H_{0}$, we used the values of Tab. I, and we inserted back the explicit numerical value of the light velocity so that $C_{P 0}$ and $C_{V 0}$ are expressed in units $\frac{J}{K}$. The specific heats are expressed in powers of $10^{52}$.

satisfies the condition $w \propto \rho^{-1}$. Then, by using Eq. (14), one gets

$$
\begin{aligned}
& \rho(z)=\rho_{0}\left[1-\kappa(1+z)^{3}\right], \\
& w(z)=-\frac{1}{1-\kappa(1+z)^{3}},
\end{aligned}
$$

where $\kappa$ is an integrating constant. Notice that the first equation of (18) can be interpreted as a thermodynamic correction to the collisionless dust term.

Under this hypothesis, the Hubble rate of the corresponding cosmological model is therefore

$$
H=H_{0} \sqrt{\frac{\kappa}{\kappa-1}(1+z)^{3}+\frac{1}{1-\kappa}},
$$

which generalizes the $\Lambda$ CDM model [55]. In fact, Eq. (19) reduces to $\Lambda$ CDM in the limiting case $\kappa \rightarrow 1-\frac{1}{\Omega_{\Lambda}}$, where $\Omega_{\Lambda}$ is the cosmological constant density. In addition, we notice that $P<0$, by requiring $\dot{H}>0$.

It is important to mention that although the $\Lambda \mathrm{CDM}$ model is recovered as a special case, the interpretation of our DE term, i.e. $\Omega_{X} \equiv \frac{1}{1-\kappa}$, is not that of the vacuum energy. Its origin is a consequence of the vanishing of the specific heat $C_{P}$.

In order to fix the theoretical limits on $\kappa$ and to predict its sign, we can find the corresponding temperature evolution. So, since the universe enthalpy is a constant and the universe evolves adiabatically, for a perfect gas we have that $P=-\rho\left(\mathrm{C}_{V}-\mathrm{C}_{P}\right) T$, and $P=\tilde{P} \rho^{\frac{c_{P}}{c_{V}}}$, where $\tilde{P}$ is an integration constant. The corresponding EoS is therefore $w=-C_{V} T$ with an explicit temperature evolution forced to be

$$
T=T_{0}(1+z)^{3 \gamma \kappa} e^{-\gamma\left[1-\frac{1}{(1+z)^{3}}\right]},
$$

with $\gamma \equiv \frac{H_{0}^{3} V_{0}}{8 \pi G} \Omega_{X}$ and $T_{0}$ the temperature at our time, i.e. $T_{0}=2.73 \mathrm{~K}$.

The temperature evolution, found under the hypothesis of $C_{P}=0$ and $C_{V}<0$, agrees with the thermodynamic results obtained in [43]. In addition, we must assume $\kappa<0$, in order to reproduce the temperature evolution of nonrelativistic particles in standard cosmology. Thus, according to Eqs. (18), the sign of $\kappa$ is able to naturally predict an accelerated universe, without imposing any further condition on the Einstein equations. This requirement seems to confirm the need of extending the $\Lambda$ CDM model as a limiting case of a more general paradigm and candidates our approach to be a serious alternative to the $\Lambda$ CDM paradigm.

\section{CONCLUSIONS}

In this work, we use standard definitions of classical thermodynamics to define the specific heats of the universe on a cosmological background given by the FLRW spacetime and a perfect fluid as the source of the gravitational interaction. We use the weak energy conditions to prove the positivity of the internal energy and the enthalpy, the thermodynamic potentials that define the specific heat at constant volume and pressure, respectively.

We propose to use cosmography to relate the specific heats with quantities that can be evaluated from observational data. In particular, we established the relationship between the specific heat and the cosmographic parameters corresponding to acceleration $q_{0}$ and jerk $j_{0}$. Since the explicit value of the cosmographic parameters can be determined 
from the observed values for the redshift and the luminosity distance, we show that today the specific heat at constant volume $C_{V}$ is negative and the specific heat at constant pressure $C_{P}$ is close to zero. These results are in agreement with the $\Lambda \mathrm{CDM}$ model in which $\mathrm{C}_{P}$ is zero and does not evolve in time.

We also analyzed the most general cosmological model which can be derived from the assumption $C_{P}=0$. It turns out that the $\Lambda \mathrm{CDM}$ model is contained in this generalized model as a special case, and that the known problems of the $\Lambda$ CDM paradigm can be overcome in a canonical way. In fact, although we consider Einstein's equations without cosmological constant, the model presented here leads to an emerging cosmological constant, which, therefore, has no reason to be associated to the vacuum energy. This fact naturally avoids the fine-tuning problem, because in our model there is no cosmological constant in the Einstein equations that should be compared with the predictions of Quantum Field Theory. Concerning the coincidence problem, since we assume an evolutionary barotropic factor $w=w(z)$, the corresponding value at $z=0$ is fixed my the matter evolution itself. The fixing of this initial condition naturally avoids the coincidence problem; in fact, it is not casual that we live in a time in which the energy density, which explains the acceleration of the universe, has a magnitude comparable with $\Omega_{m}$.

Thus, we conclude that it represents a viable alternative to the $\Lambda$ CDM approach. However, it is essential to perform additional tests to show that it can be used as a realistic cosmological alternative. In particular, in the future we expect to perform a detailed analysis of the consequences of our approach on structure formation and on the evolution of the universe through different epochs.

\section{Acknowledgements}

We are grateful to S. Capozziello, D. Pavón and A. Bravetti for fruitful discussions. This work was supported in part by DGAPA-UNAM, grant No. 106110, and Conacyt, grant No. 166391.

[1] S. Perlmutter, et al., ApJ, 483, 565, (1997); S. Perlmutter, et al., Nature, 391, 51, (1998).

[2] A. G. Riess et al., Astr. Phys. Jour., 116, 1009, (1998).

[3] S. Perlmutter et al., Astr. Phys. Jour., 517, 565, (1999).

[4] R. Rebolo et al., Mon. Not. Roy. Astr. Soc., 353, 747, (2004)

[5] A. C. Pope et al., Astr. Phys. Jour., 607, 655, (2004)

[6] P. McDonald et al., eprint: ArXiv:astro-ph/0405013. (2004).

[7] J. L. Cervantes-Cota, G. Smoot, AIP Conf. Proc. Vol., 1396, 28-52, (2011).

[8] M. Li, X.D. Li, S. Wang, Y. Wang, Com. Theor. Phys., 56, 525-604, (2011).

[9] T. Padmanabhan, Phys. Rept., 380, 235, (2003).

[10] M. Tegmark et al. (SDSS), Phys. Rev. D, 74, 123507, (2006).

[11] W. J. Percival et al., Mon. Not. Roy. Astr., Soc., 381, 1053, (2007).

[12] O. Luongo, H. Quevedo, ArXiv: gr-qc/1005.4532, (2010).

[13] S. Weinberg, Cosmology, Oxford Univ. Press, Oxford, (2008).

[14] S.M. Carroll, W.H. Press, E.L. Turner, ARAA, 30, 499, (1992).

[15] V. Sahni, A. Starobinski, Int. J. Mod. Phys. D, 9, 373, (2000).

[16] M. Tegmark et al., Phys. Rev. D, 69, 103501, (2003).

[17] U. Seljak et al., Phys. Rev. D, 71, 103515, (2005).

[18] S. M. Carroll, Liv. Rev. Rel., 4, 1, (2001).

[19] S. Tsujikawa, eprint: ArXiv:gr-qc/1004.1493, (2010).

[20] J. E. Copeland, M. Sami, S. Tsujikawa, Int. J. Mod. Phys. D, 15, 1753-1936, (2006).

[21] S. Weinberg, Rev. Mod. Phys., 61, 1, (1989).

[22] R. Durrer, R. Maartens, Gen. Rel. Grav., 40, 301-328, (2008); V. Sahni, A. A. Starobinsky, Int. J. Mod. Phys. D, 9, 373, (2000); V. Sahni, Lect. Not. Phys., 653, 141, (2004).

[23] P. J. E. Peebles, B. Ratra, Rev. Mod. Phys., 75, 559, (2003); C. Wetterich, Nucl. Phys. B., 302, 668, (1988); Y. Fujii, T. Nishioka, Phys. Rev. D, 42, 361, (1990).

[24] S. M. Carroll, Phys. Rev. Lett., 81, 3067, (1998); E. J. Copeland, A. R. Liddle, D. Wands, Phys. Rev. D, 57, 4686, (1998).

[25] R. R. Caldwell, R. Dave and P. J. Steinhardt, Phys. Rev. Lett., 80, 1582, (1998); I. Zlatev, L. M. Wang, P. J. Steinhardt, Phys. Rev. Lett., 82, 896, (1999); P. J. Steinhardt, L. M. Wang, I. Zlatev, Phys. Rev. D, 59, 123504, (1999).

[26] T. Padmanabhan, Phys. Rept., 380, 235, (2003).

[27] H. Quevedo, J. Math. Phys. 48013506 (2007).

[28] A. Vazquez, H. Quevedo, A. Sanchez, J. Geom. Phys., 60, 1942, (2010).

[29] G. Montani, Class. Quant. Grav., 18, 193-203, (2001).

[30] I. Prigogine, Thermodynamics of Irreversible Processes, (Wiley New York), (1961).

[31] I. Prigogine et al., Proc. Nat. Acad., (1988). 
[32] I. Prigogine et al., Gen. Rel. and Grav., 21, 767, (1989).

[33] K. Desikan, Gen. Rel. and Grav., 29, 4, 435, (1997).

[34] A. Aviles, A. Bastarrachea, L. Campuzano and H. Quevedo, Phys. Rev. D, 86, 063508, (2012).

[35] C. Tsallis, Non-extensive Statistical Mechanics and Applications, (eds. S. Abe and Y. Okamoto), Springer, 3-98, (2001).

[36] S. Weinberg, Gravitation and Cosmology: Principles and applications of the general theory of relativity, (Wiley, New York), (1972).

[37] M. Visser, C. Cattoën, Class. Quant. Grav., 24, 5985, (2007)

[38] M. Visser, C. Cattoën, ArXiv:gr-qc/0703122, (2007).

[39] U. Alam, V. Sahni, T. D. Saini, and A. A. Starobinsky, Mon. Not. Roy. Astr., Soc., 344, 1057, (2003).

[40] O. Luongo, Mod. Phys. Lett. A, 26, 20, (2011); A. Aviles, L. Bonanno, O. Luongo, H. Quevedo, Phys. Rev. D, 84, 103520, (2011); A. Aviles, C. Gruber, O. Luongo, H. Quevedo, Arxiv: 1204.2007, (2011).

[41] V. Sahni, T. D. Saini and A. A. Starobinsky, JETP Lett., 77, 201, (2003).

[42] U. Alam, V. Sahni, T. D. Saini, and A. A. Starobinsky, Mon. Not. Roy. Astr., Soc., 344, 1057, (2003).

[43] R. Silva, R. S. Goncalves, J. S. Alcaniz, H. B. Silva, Astr. and Astroph., 537, A11, (2012); J. C. Carvalho, J. S. Alcaniz, Month. Not. R. Astr. Soc., 418, 1873, (2011).

[44] J. A. S. Lima, J. S. Alcaniz, Phys. Lett. B, 600, 191, (2004); C. W. Misner, K. S. Thorne, J. A. Wheeler, Gravitation, W. H. Freeman, (1973).

[45] A. Krasinski, H. Quevedo, R. Sussman, J. Math. Phys., 38, 2602, (1997).

[46] D. Lynden-Bell and R. Wood, Mon. Not. Roy. Astr., Soc., 138, 495, (1968).

[47] D. Lynden-Bell, Physica A, 263, 1-4, (1999).

[48] T. Padmanabhan, Phys. Rep., 188, 285, (1990).

[49] B. Einarsson, Phys. Lett. A, 332, 335-344, (2004).

[50] P. K. Kundu, I. M. Cohen, Fluid Mechanics, Elsevier Acad. Press, San Diego, USA, (2004).

[51] R. G. Cai, S. P. Kim, JHEP, 0502, 050, (2005); R. G. Cai, L. M. Cao, Y. P. Hu, Class. Quant. Grav., 26, 155018, (2009); S. del Campo, I. Duran, R. Herrera, D. Pavon, Phys. Rev. D, 86, 083509, (2012).

[52] T. Jacobson, Phys. Rev. Lett., 75, 1260, (1995); C. Rovelli, arXiv:1209.0065, (2012).

[53] L. Xu, Y. Wang, Phys. Lett. B, 702, 114-120, (2011).

[54] N. Suzuki, D. Rubin, C. Lidman, G. Aldering, R. Amanullah, et al., Astrophys. J. 746, 85, (2012); E. Komatsu, et al., Astrop. J. Sup., 192, 18, (2011).

[55] O. Luongo, H. Quevedo, ArXiv:gr-qc/1104.4758, (2011); O. Luongo, H. Quevedo, Astroph. and Sp. Sci. 338, 2, 345-349, (2011). 